\title{
HPV-Mediated (p16-Positive) Oropharyngeal Cancer cM0 TNM Finding \\ v8
}

National Cancer Institute

\section{Source}

National Cancer Institute. HPV-Mediated (p16-Positive) Oropharyngeal Cancer CMO TNM

Finding v8. NCI Thesaurus. Code C132879.

HPV-mediated (p16-positive) oropharyngeal cancer without evidence of distant

metastasis. (from AJCC 8th Ed.) 